\title{
On a Periodic Time-Dependent Model of Population Dynamics with Stage Structure and Impulsive Effects
}

\author{
Kaiyuan Liu $^{1,2}$ and Lansun Chen ${ }^{2}$ \\ ${ }^{1}$ Department of Mathematics, Anshan Normal University, Anshan 114007, China \\ ${ }^{2}$ Department of Applied Mathematics, Dalian University of Technology, Dalian 116024, China
}

Correspondence should be addressed to Kaiyuan Liu, liukaiyuan_1963@sohu.com

Received 12 June 2007; Revised 5 February 2008; Accepted 20 March 2008

Recommended by Xue-Zhong He

We consider a periodic time-dependent predator-prey system with stage structure and impulsive harvesting, in which the prey has a life history that takes them through two stages, immature and mature. A set of sufficient and necessary conditions which guarantee the permanence of the system is obtained. Finally, we give a brief discussion of our results.

Copyright ( 2008 K. Liu and L. Chen. This is an open access article distributed under the Creative Commons Attribution License, which permits unrestricted use, distribution, and reproduction in any medium, provided the original work is properly cited.

\section{Introduction}

In the natural world, there are many species whose individual members have a life history that takes them through two stages, immature and mature. In particular, we have mammalian populations and some amphibious animals in mind, which exhibit these two stages. From the view point of mathematics, the description of the stage structure of the population in the life history is also an interesting problem in population dynamics. The permanence and extinction of species are significant concepts for those stage-structured population dynamical systems. Recently, stage structure models have been studied by many authors [1-3]. This is not only because they are much more simple than the models governed by partial differential equations but also because they can exhibit phenomena similar to those of partial differential models [4], and many important physiological parameters can be incorporated. Much research has been devoted to the models concerning single-species population growth with the stage structure of immature and mature [5,6]. Two species models with stage structure were investigated by Wang and Chen (1997), Magnusson (1999), Xiao and Chen (2003), as well as Cui and Song (2004). Also, Zhang, Chen, and Neumann (2000) proposed the following autonomous stage 
structure predator-prey system:

$$
\begin{aligned}
& x_{1}^{\prime}=\alpha x_{2}-r_{1} x_{1}-\beta x_{1}-\eta x_{1}^{2}-\beta_{1} x_{1} x_{3}, \\
& x_{2}^{\prime}=\beta x_{1}-r_{2} x_{2} \\
& x_{3}^{\prime}=x_{3}\left(-r+k \beta_{1} x_{1}-\eta_{1} x_{3}\right),
\end{aligned}
$$

where $\alpha, \beta, \beta_{1}, \eta, \eta_{1}, r, r_{1}, r_{2}$, and $k$ are all positive constants, $k$ is a digesting constant.

On the other hand, since biological and environmental parameters are naturally subject to fluctuation in time, the effects of a periodically varying environment are considered as important selective forces on systems in a fluctuating environment. So more realistic and interesting models should take into account the seasonality of the changing environment $[7,8]$. This motivated Cui and Y. Takeuchi (2006) to consider the following periodically nonautonomous predator-prey model with stage structure for prey:

$$
\begin{aligned}
\dot{x}_{1} & =a(t) x_{2}-b(t) x_{1}-d(t) x_{1}^{2}-p(t) \phi\left(t, x_{1}\right) x_{1} y, \\
\dot{x}_{2} & =c(t) x_{1}-f(t) x_{2}^{2} \\
\dot{y} & =y\left[-g(t)+h(t) \phi\left(t, x_{1}\right) x_{1}-q(t) y\right],
\end{aligned}
$$

in which $a(t), b(t), c(t), d(t), f(t), g(t), h(t), p(t), q(t)$, and $\phi\left(t, x_{1}\right)$ are all continuous $\omega$-periodic functions; $a(t), b(t), c(t), d(t), f(t), h(t), p(t), q(t)$ are all positive; $g(t), \phi\left(t, x_{1}\right)$ are nonnegative; $x_{1}$ and $x_{2}$ denote the density of immature and mature population (prey), respectively; and $y$ is the density of the predator that only prey on $x_{1}$ (immature prey). They provided a set of sufficient and necessary conditions to guarantee the permanence of the above system.

Systems with impulsive effects describing evolution processes are characterized by the fact that at certain moments of time, they experience a change of state abruptly. Processes of such type are studied in almost every domain of applied science. Impulsive equations $[9,10]$ have been recently used in population dynamics in relation to impulsive vaccination [11, 12], population ecology [13, 14], the chemotherapeutic treatment of disease [15], birth pulses [16], as well as the theory of the chemostat [17].

Let us assume that the predator population is affected by harvesting (e.g., fishing or hunting). Further, as we all know that the harvesting does not occur continuously, that is, the harvesting occurs in regular pulses, then let us assume that at some fixed moments, the predator population in system (1.2) is subject to a perturbation which incorporates the proportional decrease. After a perturbation at step $\tau_{k}>0(k \in \mathbf{N})$, the size of the population $y\left(\tau_{k}^{+}\right)$becomes

$$
y\left(\tau_{k}^{+}\right)=\left(1-u_{k}\right) y\left(\tau_{k}\right),
$$

where $y\left(\tau_{k}\right)$ is the size of the predator population at step $\tau_{k}$ before the impulsive perturbation, and $0<u_{k}<1$ represents the rate at which the predator is harvested.

In this article, we extend the model (1.2) to the case when the predator population is omnivorous and affected by impulsive effects, which is governed by the following system:

$$
\begin{aligned}
& \dot{x}_{1}=a(t) x_{2}-b(t) x_{1}-d(t) x_{1}^{2}-p_{1}(t) \phi_{1}\left(t, x_{1}\right) x_{1}^{2} y, \quad t \neq \tau_{k}, \\
& \dot{x}_{2}=c(t) x_{1}-f(t) x_{2}^{2}-p_{2}(t) \phi_{2}\left(t, x_{2}\right) x_{2}^{2} y, \quad t \neq \tau_{k},
\end{aligned}
$$




$$
\begin{aligned}
\dot{y} & =y\left[g(t)+\sum_{i=1}^{2} h_{i}(t) \phi_{i}\left(t, x_{i}\right) x_{i}^{2}-q(t) y(t)\right], \quad t \neq \tau_{k}, \\
\Delta y(t) & =-u_{k} y(t), \quad t=\tau_{k}, k \in \mathbf{N}
\end{aligned}
$$

with the initial value conditions

$$
x_{1}(0)=x_{10} \geq 0, \quad x_{2}(0)=x_{20} \geq 0, \quad y(0)=y_{0} \geq 0,
$$

in which $x_{1}$ and $x_{2}$ are the densities of immature and mature prey, respectively; and $y$ is the density of the predator that can prey on $x_{1}$ and $x_{2}$. When its favorite food is severely scarce, population $y$ can eat other resources: $\Delta y(t)=y\left(t^{+}\right)-y(t)$. Also, there exists a positive integer $q$ such that

$$
\begin{gathered}
u_{k+q}=u_{k}, \quad \tau_{k+q}=\tau_{k}+\omega, \\
0=\tau_{0}<\tau_{1}<\tau_{2}<\cdots<\tau_{k}<\tau_{k+1}<\cdots, \quad k \in \mathbf{N} .
\end{gathered}
$$

Here, $x_{i}^{2} \phi_{i}\left(t, x_{i}\right)$, the number of the prey $x_{i}$ consumed per predator in unit time, is called the predator functional response. We assume that there exists a positive constant $L$ such that

$$
0<\phi_{i}\left(t, x_{i}\right)<L ; \quad \frac{\partial}{\partial x_{i}}\left(x_{i}^{2} \phi_{i}\left(t, x_{i}\right)\right) \geq 0 \quad \text { for } x_{i}>0, i=1,2 .
$$

The last condition in (1.7) implies that as the prey population increases, the consumption rate of prey consumed per predator increases. The birth rate of the immature prey population is proportional to the existing mature prey population with a proportionality function $a(t)$. For the immature prey population, the death rate is proportional to the existing immature prey population with a proportionality function $b(t)$. The variable parameter $d(t)$ represents that the immature prey population is density restriction. The transition rate from immature individuals to the mature individuals is assumed to be proportional to the existing immature population with proportionality coefficient $c(t)$. The death rate of the mature population is of a logistic nature with proportionality coefficient $f(t)$. Also, $p_{i}(t)$ and $h_{i}(t)(i=1,2)$ are the coefficients that relate to conversion rates of the immature and, respectively, mature prey biomass into predator biomass. The coefficients in (1.4) are all continuous $\omega$-periodic for $t \geq 0$. In fact, $a(t)$, $b(t), c(t), d(t), f(t), p_{i}(t), h_{i}(t)$, and $q(t)$ are all strictly positive, and $\phi_{i}\left(t, x_{i}\right)$ is nonnegative $(i=1,2)$.

The organization of this paper is as follows. In Section 2, we provide some preliminary results which will be useful. In Section 3, we investigate the permanence and extinction of system (1.4) by using analysis technique. In the last section, we give a biological example and a brief discussion of our result.

\section{Preliminary results}

Before stating and proving our main results, we give the following definitions, notations, and lemmas which will be useful in the following section.

Let $\mathbf{R}_{+}=[0, \infty), \mathbf{R}_{+}^{n}=\left\{\left(x_{1}, \ldots, x_{n}\right): x_{i}>0, i=1, \ldots, n\right\}$ and $\alpha(t)$ be a continuous $\omega$-periodic function defined on $[0,+\infty)$, then we set

$$
\mathbf{A}_{\omega}(\alpha)=\omega^{-1} \int_{0}^{\omega} \alpha(t) d t, \quad \alpha^{U}=\max _{t \in[0, \omega]} \alpha(t), \quad \alpha^{L}=\min _{t \in[0, \omega]} \alpha(t) .
$$


Lemma 2.1. System (1.4) is dissipative.

Proof. Define a function $V\left(t, x_{1}, x_{2}, y\right)$ such that

$$
V\left(t, x_{1}, x_{2}, y\right)=h\left(x_{1}(t)+x_{2}(t)\right)+p y(t)
$$

in which $h=\max _{t \in[0, \omega]}\left\{h_{1}(t), h_{2}(t)\right\}, p=\min _{t \in[0, \omega]}\left\{p_{1}(t), p_{2}(t)\right\}$. After a simple computations, we have

$$
\left.D^{+} V\right|_{(4)}+\bar{w} V<\left(h a^{U}+\bar{w}\right) x_{2}-h f^{L} x_{2}^{2}+\left(h c^{U}+\bar{w}-h b^{L}\right) x_{1}-h d^{L} x_{1}^{2}+\left(\bar{w}+p g^{U}\right) y-p q^{L} y^{2}
$$

in which $\bar{w}$ is a positive constant. Obviously, the right-hand side of the above inequality is bounded above for all $\left(x_{1}(t), x_{2}(t), y(t)\right) \in R_{+}^{3}$. Hence,

$$
\left.D^{+} V\right|_{(4)}+\bar{w} V<\lambda,
$$

where $\lambda$ is a positive constant. When $t=\tau_{k}$, we get

$$
V\left(\tau_{k}^{+}\right) \leq V\left(\tau_{k}\right)
$$

According to Lemma 2.2 in [9, page 23$]$, we derive that

$$
V(t)=V(0) e^{-\bar{w} t}+\int_{0}^{t} \lambda e^{-\bar{w}(t-s)} d s<\frac{2 \lambda}{\bar{w}}, \quad \text { as } t \longrightarrow \infty,
$$

which implies that system (1.4) is dissipative. This completes the proof.

Next, we consider the following two subsystems of system (1.4):

$$
\begin{aligned}
\dot{x}_{1} & =a(t) x_{2}-b(t) x_{1}-d(t) x_{1}^{2}, \\
\dot{x}_{2} & =c(t) x_{1}-f(t) x_{2}^{2}, \\
\dot{y} & =y[g(t)-q(t) y(t)], \quad t \neq \tau_{k}, \\
\Delta y(t) & =-u_{k} y(t), \quad t=\tau_{k}, \quad k \in \mathbf{N} .
\end{aligned}
$$

Lemma 2.2 (see [18]). The system (2.7) has a positive w-periodic solution $\left(x_{1}^{*}(t), x_{2}^{*}(t)\right)$ which is globally asymptotically stable with respect to $\mathbf{R}_{+}^{2}$.

Lemma 2.3. If the following conditions

$$
\begin{gathered}
\mathbf{A}_{\omega}(g)>0, \\
\prod_{k=1}^{q}\left(1-u_{k}\right)>\exp \left(-\omega \mathbf{A}_{\omega}(g)\right)
\end{gathered}
$$


hold, then system (2.8) has a unique w-periodic solution:

$$
\begin{aligned}
y^{*}(t)= & \left(\frac{\prod_{0<\tau_{k}<t}\left(1 /\left(1-u_{k}\right)\right) \exp \left(-\int_{0}^{t} g(\xi) d \xi\right) \int_{0}^{\omega} \prod_{s \leq \tau_{k}<\omega}\left(1 /\left(1-u_{k}\right)\right) \exp \left(-\int_{s}^{\omega} g(\xi) d \xi\right) q(s) d s}{1-\prod_{k=1}^{q}\left(1 /\left(1-u_{k}\right)\right) \exp \left(-\int_{0}^{\omega} g(\xi) d \xi\right)}\right. \\
& \left.+\int_{0}^{t} \prod_{s \leq \tau_{k}<t} \frac{1}{1-u_{k}} \exp \left(-\int_{s}^{t} g(\xi) d \xi\right) q(s) d s\right)^{-1}
\end{aligned}
$$

and for every solution $y(t)$ of system (2.8),

$$
\left|y(t)-y^{*}(t)\right| \longrightarrow 0 \text { as } t \longrightarrow \infty
$$

Proof. Let $y(t)=1 / z(t)$ and obtain the linear nonhomogeneous impulsive equation

$$
\begin{aligned}
\dot{z}(t) & =-g(t) z(t)+q(t), \quad t \neq \tau_{k}, \\
z\left(t^{+}\right) & =\frac{1}{1-u_{k}} z(t), \quad t=\tau_{k} .
\end{aligned}
$$

Let $W(t, s)=\prod_{s \leq \tau_{k}<t}\left(1 /\left(1-u_{k}\right)\right) \exp \left(-\int_{s}^{t} g(\xi) d \xi\right)$ be the Cauchy matrix for the relevant homogeneous equation, then the solution of (2.12) has the form

$$
z(t)=W(t, 0) z(0)+\int_{0}^{t} W(t, s) q(s) d s
$$

The solution $z(t)$ will be $\omega$-periodic if $z(\omega)=z(0)$, or if

$$
(1-W(\omega, 0)) z(0)=\int_{0}^{\omega} W(\omega, s) q(s) d s .
$$

In view of conditions (2.9), (2.14) has a unique solution

$$
z(0)=\frac{\int_{0}^{\omega} W(\omega, s) q(s) d s}{1-W(\omega, 0)} .
$$

Then, (2.13) has a unique $\omega$-periodic solution

$$
z^{*}(t)=\frac{W(t, 0) \int_{0}^{\omega} W(\omega, s) q(s) d s}{1-W(\omega, 0)}+\int_{0}^{t} W(t, s) q(s) d s .
$$

Hence, (2.8) has a unique $\omega$-periodic solution

$$
\begin{aligned}
y^{*}(t)= & \left(\frac{W(t, 0) \int_{0}^{\omega} W(\omega, s) q(s) d s}{1-W(\omega, 0)}+\int_{0}^{t} W(t, s) q(s) d s\right)^{-1} \\
= & \left(\frac{\prod_{0 \leq \tau_{k}<t}\left(1 /\left(1-u_{k}\right)\right) \exp \left(-\int_{0}^{t} g(\xi) d \xi\right) \int_{0}^{\omega} \prod_{s \leq \tau_{k}<\omega}\left(1 /\left(1-u_{k}\right)\right) \exp \left(-\int_{s}^{\omega} g(\xi) d \xi\right) q(s) d s}{1-\prod_{k=1}^{q}\left(1 /\left(1-u_{k}\right)\right) \exp \left(-\int_{0}^{\omega} g(\xi) d \xi\right)}\right. \\
& \left.+\int_{0}^{t} \prod_{s \leq \tau_{k}<t} \frac{1}{1-u_{k}} \exp \left(-\int_{s}^{t} g(\xi) d \xi\right) q(s) d s\right)^{-1} .
\end{aligned}
$$


From (2.12), we derive that

$$
\left(z(t)-z^{*}(t)\right)^{\prime}=-g(t)\left(z(t)-z^{*}(t)\right), \quad \tau_{k}<t \leq \tau_{k+1} .
$$

Let

$$
\mathcal{\kappa} \doteq \prod_{k=1}^{q} \frac{1}{1-u_{k}} \exp \left(-\int_{0}^{\omega} g(t) d t\right)
$$

Since conditions (2.9) hold, we note that $0<\kappa<1$. Then, from Lemma 2.1, the solution of (2.8) is governed by

$$
\begin{aligned}
\left|y(t)-y^{*}(t)\right| & \leq \frac{2 \lambda}{\bar{w}} y^{*^{u}}\left|z\left(0^{+}\right)-z^{*}\left(0^{+}\right)\right| \prod_{0<\tau_{k}<t} \frac{1}{1-u_{k}} \exp \left(-\int_{0}^{t} g(s) d s\right), \\
& \leq \frac{2 \lambda}{\bar{w}} y^{*^{u}}\left|z\left(0^{+}\right)-z^{*}\left(0^{+}\right)\right| \kappa^{[t / \omega]+1} \longrightarrow 0 \text { as } t \longrightarrow \infty,
\end{aligned}
$$

in which $\lambda$ and $\bar{w}$ are defined in Lemma 2.1. This completes the proof.

\section{Permanence and extinction of system}

Theorem 3.1. System (1.4) is permanent if and only if

$$
\begin{gathered}
\mathbf{A}_{\omega}\left(g+\sum_{i=1}^{2} h_{i} \phi_{i}\left(t, x_{i}^{*}\right) x_{i}^{*^{2}}\right)>0, \\
\prod_{k=1}^{q}\left(1-u_{k}\right)>\exp \left(-\omega \mathbf{A}_{\omega}\left(g+\sum_{i=1}^{2} h_{i} \phi_{i}\left(t, x_{i}^{*}\right) x_{i}^{*^{2}}\right)\right),
\end{gathered}
$$

in which $\left(x_{1}^{*}(t), x_{2}^{*}(t)\right)$ is the positive w-periodic solution of system (2.7).

We need the following lemmas to prove Theorem 3.1.

Lemma 3.2. There exist positive constants $M_{x}$ and $M_{y}$ such that

$$
\limsup _{t \rightarrow \infty} x_{i}(t) \leq M_{x} \quad(i=1,2), \quad \limsup _{t \rightarrow \infty} y(t) \leq M_{y} .
$$

Proof. In fact, from Lemmas 2.1 and 2.3, the proof of the lemma is obvious. This completes the proof.

Lemma 3.3. There exists a positive constant $\rho_{x}\left(\rho_{x}<M_{x}\right)$ such that

$$
\liminf _{t \rightarrow \infty} x_{i}(t) \geq \rho_{x} \quad(i=1,2)
$$


Proof. From Lemma 3.2, one notes that there exists a positive constant $T_{0}$ such that

$$
0<y(t) \leq M_{y} \text { for } t \geq T_{0}
$$

we then have

$$
\begin{gathered}
\dot{x}_{1} \geq a(t) x_{2}-b(t) x_{1}-\left(d(t)+p_{1}(t) L M_{y}\right) x_{1}^{2} \\
\dot{x}_{2} \geq c(t) x_{1}-\left(f(t)+p_{2}(t) L M_{y}\right) x_{2}^{2}
\end{gathered}
$$

for $t \geq T_{0}$. According to Lemma 2.2, the following auxiliary equations:

$$
\begin{aligned}
& \dot{u}_{1}=a(t) u_{2}-b(t) u_{1}-\left(d(t)+p_{1}(t) L M_{y}\right) u_{1}^{2} \\
& \dot{u}_{2}=c(t) u_{1}-\left(f(t)+p_{2}(t) L M_{y}\right) u_{2}^{2}
\end{aligned}
$$

has a globally asymptotically stable $\omega$-periodic solution $\left(u_{1}^{*}(t), u_{2}^{*}(t)\right)$. Let $\left(u_{1}(t), u_{2}(t)\right)$ be the solution of (3.6) with $\left(u_{1}(0), u_{2}(0)\right)=\left(x_{1}(0), x_{2}(0)\right)$. By the vector comparison theorem [19], we obtain

$$
x_{i}(t) \geq u_{i}(t), \quad i=1,2 \forall t \geq 0 .
$$

According to the global asymptotic stability of $\left(u_{1}^{*}(t), u_{2}^{*}(t)\right)$, for any positive constant $\varepsilon(\leq$ $\left.\min _{t \in[0, \omega]}\left\{u_{i}^{*}(t) / 3, i=1,2\right\}\right)$, there exists a $T_{1}\left(>T_{0}\right)$ such that for all $t \geq T_{1}$,

$$
\left|u_{i}(t)-u_{i}^{*}(t)\right|<\varepsilon, \quad i=1,2 .
$$

Hence, for all $t \geq T_{1}$, we derive that

$$
u_{i}(t) \geq u_{i}^{*}(t)-\varepsilon, \quad i=1,2 .
$$

Let

$$
\rho_{x}=\max _{t \in[0, \omega]}\left\{\frac{u_{i}^{*}(t)}{2}, i=1,2\right\},
$$

then

$$
x_{i}(t) \geq \rho_{x}, \quad i=1,2 .
$$

Consequently,

$$
\liminf _{t \rightarrow \infty} x_{i}(t) \geq \rho_{x}, \quad i=1,2 .
$$

This completes the proof.

Lemma 3.4. Suppose that (3.1) holds, then there exists a positive constant $\rho_{y}\left(\rho_{y}<M_{y}\right)$ such that

$$
\limsup _{t \rightarrow \infty} y(t) \geq \rho_{y}
$$


Proof. In view of (3.1), we can choose a positive constant $\varepsilon_{0}$ such that

$$
\begin{gathered}
\mathbf{A}_{\omega}\left(\psi_{\varepsilon_{0}}(t)\right)>0 \\
\prod_{k=1}^{q}\left(1-u_{k}\right)>\exp \left(-\omega \mathbf{A}_{\omega}\left(\psi_{\varepsilon_{0}}(t)\right)\right)
\end{gathered}
$$

in which

$$
\psi_{\varepsilon_{0}}(t)=g(t)+\sum_{i=1}^{2} h_{i}(t) \phi_{i}\left(t,\left(x_{i}^{*}(t)-\varepsilon_{0}\right)\right)\left(x_{i}^{*}(t)-\varepsilon_{0}\right)^{2}-q(t) \varepsilon_{0}
$$

Consider the following system with a positive parameter $\mu$ :

$$
\begin{aligned}
& \dot{x}_{1}=a(t) x_{2}-b(t) x_{1}-\left[d(t)+L \mu p_{1}(t)\right] x_{1}^{2}, \\
& \dot{x}_{2}=c(t) x_{1}-\left[f(t)+L \mu p_{2}(t)\right] x_{2}^{2} .
\end{aligned}
$$

By Lemma 2.2, system (3.16) has a positive $\omega$-periodic solution $\left(x_{1 \mu}^{*}(t), x_{2 \mu}^{*}(t)\right)$, which is globally asymptotically stable. Let $\left(x_{1 \mu}(t), x_{2 \mu}(t)\right)$ be the solution of (3.16) with initial condition $x_{i \mu}(0)=x_{i}^{*}(0), i=1,2$, where $\left(x_{1}^{*}(t), x_{2}^{*}(t)\right)$ is the positive periodic solution of (2.7). Hence, for the above $\varepsilon_{0}$, there exists $T_{2}>T_{1}$ such that

$$
\left|x_{i \mu}(t)-x_{i \mu}^{*}(t)\right|<\frac{\varepsilon_{0}}{4}
$$

for $t \geq T_{2}, i=1,2$. According to the continuity of the solution in the parameter $\mu$, we have $x_{i \mu}(t) \rightarrow x_{i}^{*}(t)(i=1,2)$ uniformly in $\left[T_{2}, T_{2}+\omega\right]$ as $\mu \rightarrow 0$. Hence, for $\varepsilon_{0}>0$, there exists $\mu_{0}=\mu_{0}\left(\varepsilon_{0}\right)\left(0<\mu_{0}<\varepsilon_{0}\right)$ such that

$$
\left|x_{i \mu}(t)-x_{i}^{*}(t)\right|<\frac{\varepsilon_{0}}{4}, \quad 0 \leq \mu \leq \mu_{0},
$$

$t \in\left[T_{2}, T_{2}+\omega\right], i=1,2$. Thus, from (3.17) and (3.18), we get

$$
\left|x_{i \mu}^{*}(t)-x_{i}^{*}(t)\right|<\frac{\varepsilon_{0}}{2}, \quad 0 \leq \mu \leq \mu_{0},
$$

$t \in\left[T_{2}, T_{2}+\omega\right], i=1,2$. Since $x_{i \mu}^{*}(t)$ and $x_{i}^{*}(t)$ are all $\omega$-periodic, we have

$$
\left|x_{i \mu}^{*}(t)-x_{i}^{*}(t)\right|<\frac{\varepsilon_{0}}{2}, \quad 0 \leq \mu \leq \mu_{0},
$$

$t \geq 0, i=1,2$.

Choose a constant $\mu_{1}\left(0<\mu_{1}<\mu_{0}, \mu_{1}<\varepsilon_{0}\right)$, from (3.20), we derive

$$
x_{i \mu_{1}}^{*}(t) \geq x_{i}^{*}(t)-\frac{\varepsilon_{0}}{2}, \quad t \geq 0, i=1,2 .
$$

Suppose that (3.13) is not true, then for the above $\varepsilon_{0}$, there exists a $v \in \mathbf{R}_{+}^{3}$ such that

$$
\limsup _{t \rightarrow \infty} y(t)<\mu_{1},
$$


where $\left(x_{1}(t), x_{2}(t), y(t)\right)$ is the solution of (1.4) with the initial condition $\left(x_{10}, x_{20}, y_{0}\right)=v$. So there exists a constant $T_{3}\left(>T_{2}\right)$ such that

$$
y(t)<\mu_{1}, \quad t \geq T_{3},
$$

then we derive that

$$
\begin{gathered}
\dot{x}_{1} \geq a(t) x_{2}-b(t) x_{1}-\left(d(t)+L \mu_{1} p_{1}(t)\right) x_{1}^{2}, \\
\dot{x}_{2} \geq c(t) x_{1}-\left(f(t)+L \mu_{1} p_{2}(t)\right) x_{2}^{2}
\end{gathered}
$$

for $t \geq T_{3}$. Let $\left(x_{1 \mu_{1}}, x_{2 \mu_{1}}\right)$ be the solution of (3.16) with $\mu=\mu_{1}$ and $\left(x_{1 \mu_{1}}\left(T_{3}\right), x_{2 \mu_{1}}\left(T_{3}\right)\right)=$ $\left(x_{1}\left(T_{3}\right), x_{2}\left(T_{3}\right)\right)$, then by the vector comparison theorem, we obtain

$$
x_{i}(t) \geq x_{i \mu_{1}}(t), \quad i=1,2,
$$

$t \geq T_{3}$. By the global asymptotic stability of $\left(x_{1 \mu_{1}}^{*}(t), x_{2 \mu_{1}}^{*}(t)\right)$, for the given $\varepsilon_{0}>0$, there exists $T_{4}>T_{3}$ such that

$$
x_{i \mu_{1}}(t)>x_{i \mu_{1}}^{*}(t)-\frac{\varepsilon_{0}}{2}, \quad t \geq T_{4}, i=1,2,
$$

and hence, by (3.21), we get

$$
x_{i}(t)>x_{i}^{*}(t)-\varepsilon_{0}, \quad t \geq T_{4}, i=1,2 .
$$

Since $0<y(t)<\mu_{1}<\varepsilon_{0}$ together with (1.4) and (1.7), we have

$$
\begin{gathered}
\dot{y} \geq y\left[g(t)+\sum_{i=1}^{2} h_{i}(t) \phi_{i}\left(t,\left(x_{i}^{*}(t)-\varepsilon_{0}\right)\right)\left(x_{i}^{*}(t)-\varepsilon_{0}\right)^{2}-q(t) \varepsilon_{0}\right], \quad t \neq \tau_{k}, \\
y\left(t^{+}\right)=\left(1-u_{k}\right) y(t), \quad t=\tau_{k}, k \in \mathbf{N} .
\end{gathered}
$$

Hence, it follows from Lemma 2.2 in [9, page 23] that

$$
y(t) \geq y_{0} \prod_{0<\tau_{k}<t}\left(1-u_{k}\right) \exp \left(\int_{0}^{t} \psi_{\varepsilon_{0}}(s) d s\right),
$$

that is,

$$
y(t) \geq y_{0}\left[\prod_{0<\tau_{k}<\omega}\left(1-u_{k}\right) \exp \left(\omega \mathbf{A}_{\omega}\left(\psi_{\varepsilon_{0}}\right)\right)\right]^{[t / \omega]} .
$$

By (3.14), we know that $y(t) \rightarrow \infty$ as $t \rightarrow \infty$, which leads to a contradiction. This completes the proof.

Lemma 3.5. Assume that (3.1) holds, then there exists a positive constant $\delta_{y}\left(\delta_{y}<M_{y}\right)$ such that any solution $\left(x_{1}, x_{2}, y\right)$ of system (1.4) with initial conditions satisfies

$$
\liminf _{t \rightarrow \infty} y(t) \geq \delta_{y}
$$


Proof. Suppose that (3.31) is not true, there must exists a time sequence $\left\{t_{t_{k}}^{(\widehat{k})}\right\}_{k=1}^{\infty} \subset \mathbf{R}_{+}, \widehat{k} \in \mathbf{Z}$ such that

$$
\liminf _{t_{k}^{(\hat{k})} \rightarrow \infty} y\left(t_{k}^{\widehat{k}}\right)<\frac{\rho_{y}}{(\widehat{k}+1)^{2}}
$$

and by Lemma 3.4, we have $\lim \sup _{t_{k}^{(\hat{k})} \rightarrow \infty} y\left(t_{k}^{(\widehat{k})}\right) \geq \rho_{y}, \widehat{k}=1,2, \ldots$ Hence, for each $\widehat{k}$, we choose two time sequences $\left\{s_{q}^{(\widehat{k})}\right\}$ and $\left\{t_{q}^{(\widehat{k})}\right\}$, satisfying $0<s_{1}^{(\hat{k})}<t_{1}^{(\widehat{k})}<s_{2}^{(\hat{k})}<t_{2}^{(\hat{k})}<\cdots<s_{q}^{(\hat{k})}<t_{q}^{(\widehat{k})}<\cdots$ and $s_{q}^{(\hat{k})} \rightarrow \infty$ as $q \rightarrow \infty$, as well as

$$
\begin{aligned}
& y\left(s_{q}^{(\widehat{k})}\right)=\frac{\rho_{y}}{\widehat{k}+1}, \quad y\left(t_{q}^{(\widehat{k})}\right)=\prod_{0<\tau_{k}<t_{q}^{(\hat{k})}}\left(1-u_{k}\right) \frac{\rho_{y}}{(\widehat{k}+1)^{2}}, \\
& \prod_{0<\tau_{k}<t_{q}^{(\hat{k})}}\left(1-u_{k}\right) \frac{\rho_{y}}{(\widehat{k}+1)^{2}}<y(t)<\frac{\rho_{y}}{\widehat{k}+1}, \quad t \in\left(s_{q}^{(\widehat{k})}, t_{q}^{(\widehat{k})}\right) .
\end{aligned}
$$

By Lemma 3.2, for a given positive integer $\widehat{k}$, there exists $\widetilde{T}^{(\hat{k})}>0$ such that $x_{i}(t) \leq M_{x}(i=1,2)$ and $y(t) \leq M_{y}$ for all $t \geq \widetilde{T}^{(\hat{k})}$. In view of $s_{q}^{(\widehat{k})} \rightarrow \infty$ as $q \rightarrow \infty$, there exists a positive integer $K_{1}^{(\widehat{k})}$ such that $s_{q}^{(\widehat{k})}>\widetilde{T}^{(\hat{k})}$ as $q \geq K_{1}^{(\widehat{k})}$. Hence, for any $t \geq \widetilde{T}^{(\widehat{k})}$, we have

$$
\begin{gathered}
\dot{y} \geq y\left(-|g(t)|-M_{y} q(t)\right), \quad t \neq \tau_{k}, \\
y\left(t^{+}\right)=\left(1-u_{k}\right) y(t), \quad t=\tau_{k}, \quad k \in \mathbf{N} .
\end{gathered}
$$

Integrating the above inequality from $s_{q}^{(\hat{k})}$ to $t_{q}^{(\widehat{k})}$, for any $q \geq K_{1}^{(\widehat{k})}$, then we have

$$
y\left(t_{q}^{(\widehat{k})}\right) \geq y\left(s_{q}^{(\widehat{k})}\right) \prod_{s_{q}^{(\hat{k})}<\tau_{k}<t_{q}^{(\hat{k})}}\left(1-u_{k}\right) \exp \left(\int_{s_{q}^{(\hat{k})}}^{t_{q}^{(\hat{k})}}\left[-|g(t)|-M_{y} q(t)\right] d t\right) .
$$

Obviously, it follows from (3.33) that

$$
\int_{s_{q}^{(\widehat{k})}}^{t_{q}^{(\widehat{k})}}\left[|g(t)|+M_{y} q(t)\right] d t>\ln (\widehat{k}+1) \quad \text { for } q \geq K_{1}^{(\widehat{k})} .
$$

Hence, in view of the periodicity of $g(t)$ and $q(t)$, we get

$$
t_{q}^{(\widehat{k})}-s_{q}^{(\widehat{k})} \longrightarrow \infty \quad \text { as } \widehat{k} \longrightarrow \infty, q \geq K_{1}^{(\widehat{k})} .
$$

By (3.14), (3.33), and (3.38), there are positive constants $T$ and $N_{0}$ such that

$$
\begin{gathered}
y\left(s_{q}^{(\widehat{k})}\right)=\prod_{0<\tau_{k} \leq s_{q}^{(\widehat{k})}}\left(1-u_{k}\right) \frac{\rho_{y}}{\widehat{k}+1}<\varepsilon_{0}, \\
t_{q}^{(\widehat{k})}-s_{q}^{(\widehat{k})}>2 T, \\
\prod_{0<\tau_{k}<\mathcal{K}}\left(1-u_{k}\right) \exp \left(\int_{0}^{\mathcal{K}} \psi_{\varepsilon_{0}}(t) d t\right)>1
\end{gathered}
$$


for $\widehat{k} \geq N_{0}$, for $q \geq K_{1}^{(\widehat{k})}$, and for all $\kappa>T$. However, (3.39) implies that

$$
y(t)<\varepsilon_{0}, \quad t \in\left[s_{q}^{(\widehat{k})}, t_{q}^{(\widehat{k})}\right]
$$

for $\widehat{k} \geq N_{0}, q \geq K_{1}^{(\widehat{k})}$. Then, we get

$$
\begin{gathered}
\dot{x}_{1} \geq a(t) x_{2}-b(t) x_{1}-\left(d(t)+L \varepsilon_{0} p_{1}(t)\right) x_{1}^{2}, \\
\dot{x}_{2} \geq c(t) x_{1}-\left(f(t)+L \varepsilon_{0} p_{2}(t)\right) x_{2}^{2} .
\end{gathered}
$$

Let $\left(x_{1 \varepsilon_{0}}, x_{2 \varepsilon_{0}}\right)$ be the solution of (3.16) with $\mu=\varepsilon_{0}$ and $\left(x_{1 \varepsilon_{0}}\left(T_{3}\right), x_{2 \varepsilon_{0}}\left(T_{3}\right)\right)=\left(x_{1}\left(T_{3}\right), x_{2}\left(T_{3}\right)\right)$, then by the vector comparison theorem, we obtain

$$
x_{i}(t) \geq x_{i \varepsilon_{0}}(t), \quad i=1,2, t \in\left[s_{q}^{(\hat{k})}, t_{q}^{(\hat{k})}\right]
$$

From $\lim _{q \rightarrow \infty} s_{q}^{(\widehat{k})}=\infty$ and Lemmas 3.2 and 3.3, we obtain that for any $\widehat{k}$, there is a $K_{2}^{(\widehat{k})}>K_{1}^{(\widehat{k})}$ such that for any $q \geq K_{2}^{(\widehat{k})}$,

$$
\rho_{x} \leq x_{i}\left(s_{q}^{(\widehat{k})}\right) \leq M_{x}, \quad i=1,2
$$

For $\mu=\varepsilon_{0}$, (3.16) has a globally asymptotically stable positive $\omega$-periodic solution $\left(x_{1 \varepsilon_{0}}^{*}(t), x_{2 \varepsilon_{0}}^{*}(t)\right)$. By the global asymptotic stability of $\left(x_{1 \varepsilon_{0}}^{*}(t), x_{2 \varepsilon_{0}}^{*}(t)\right)$, for the given $\varepsilon_{0}>0$, there exists $T_{5}>T_{4}$, and $T_{5}$ is dependent of any $\widehat{k}$ and $q$ such that

$$
x_{i \varepsilon_{0}}(t)>x_{i \varepsilon_{0}}^{*}(t)-\frac{\varepsilon_{0}}{2}, \quad t \geq T_{5}+s_{q}^{(\widehat{k})}, i=1,2, q \geq K_{2}^{(\widehat{k})},
$$

and hence, by (3.21), we get

$$
x_{i \varepsilon_{0}}(t)>x_{i}^{*}(t)-\varepsilon_{0}, \quad t \geq T_{5}+s_{q}^{(\widehat{k})}, i=1,2, q \geq K_{2}^{(\widehat{k})} .
$$

By (3.44), there is an $N_{1} \geq N_{0}$ such that $t_{q}^{(\widehat{k})}-s_{q}^{(\widehat{k})} \geq 2 T$ for all $\widehat{k} \geq N_{1}$ and $q \geq K_{2}^{(\widehat{k})}$, where $T \geq T_{5}$. Hence, from (3.44) and (3.47), we obtain

$$
x_{i}(t) \geq x_{i}^{*}(t)-\varepsilon_{0}, \quad i=1,2
$$

for all $t \in\left[T+s_{q}^{(\widehat{k})}, t_{q}^{(\widehat{k})}\right], \widehat{k} \geq N_{1}$, and $q \geq K_{2}^{(\widehat{k})}$. Since, for any $t \in\left[T+s_{q}^{(\widehat{k})}, t_{q}^{(\widehat{k})}\right], k \geq N_{1}$ and $q \geq K_{2}^{(\widehat{k})}$, by (1.4) and (3.42), we have

$$
\begin{gathered}
\dot{y} \geq y\left[g(t)+\sum_{i=1}^{2} h_{i}(t) \phi_{i}\left(t,\left(x_{i}^{*}(t)-\varepsilon_{0}\right)\right)\left(x_{i}^{*}(t)-\varepsilon_{0}\right)^{2}-q(t) \varepsilon_{0}\right], \quad t \neq \tau_{k}, t \in\left[T+s_{q}^{(\widehat{k})}, t_{q}^{(\widehat{k})}\right], \\
y\left(t^{+}\right)=\left(1-u_{k}\right) y(t), \quad t=\tau_{k}, k \in \mathbf{N} .
\end{gathered}
$$


Integrating from $T+s_{q}^{(\hat{k})}$ to $t_{q}^{(\widehat{k})}$ for any $k \geq N_{1}$ and $q \geq K_{2}^{(\hat{k})}$, we obtain

$$
y\left(t_{q}^{(\widehat{k})}\right) \geq y\left(s_{q}^{(\widehat{k})}+T\right) \prod_{s_{q}^{(\hat{k})}+T<\tau_{k}<t_{q}^{(\widehat{k})}}\left(1-u_{k}\right) \exp \left(\int_{s_{q}^{(\hat{k})}+T}^{t_{q}^{(\widehat{k})}} \psi_{\varepsilon_{0}}(t) d t\right) .
$$

Hence, by (3.34), (3.40), and (3.41), we finally derive that

$$
\begin{aligned}
\prod_{0<\tau_{k}<t_{q}^{(\hat{k})}}\left(1-u_{k}\right) \frac{\rho_{y}}{(\widehat{k}+1)^{2}} & \geq \prod_{0<\tau_{k} \leq t_{q}^{(\hat{k})}}\left(1-u_{k}\right) \frac{\rho_{y}}{(\widehat{k}+1)^{2}} \prod_{s_{q}^{(\widehat{k})}+T<\tau_{k}<t_{q}^{(\hat{k})}}\left(1-u_{k}\right) \exp \left(\int_{s_{q}^{(\widehat{k})}+T}^{t_{q}^{(\widehat{k})}} \psi_{\varepsilon_{0}}(t) d t\right) \\
& >\prod_{0<\tau_{k}<t_{q}^{(\hat{k})}}\left(1-u_{k}\right) \frac{\rho_{y}}{(\widehat{k}+1)^{2}} .
\end{aligned}
$$

This leads to a contradiction. This completes the proof.

According to Lemmas 3.2-3.5, we can directly prove the sufficient part of the theorem. Next, we are ready to show the necessity of this theorem.

Consider the case of

$$
\mathbf{A}_{\omega}\left(g+\sum_{i=1}^{2} h_{i} \phi_{i}\left(t, x_{i}^{*}\right) x_{i}^{*^{2}}\right) \leq 0
$$

it is then easy to derive that the predator population $y(t)$ must be extinct because of sterilization and impulsive harvesting. Suppose that

$$
\begin{gathered}
\mathbf{A}_{\omega}\left(g+\sum_{i=1}^{2} h_{i} \phi_{i}\left(t, x_{i}^{*}\right) x_{i}^{*^{2}}\right)>0 \\
\prod_{k=1}^{q}\left(1-u_{k}\right) \leq \exp \left(-\omega \mathbf{A}_{\omega}\left(g+\sum_{i=1}^{2} h_{i} \phi_{i}\left(t, x_{i}^{*}\right) x_{i}^{*^{2}}\right)\right) .
\end{gathered}
$$

We will show that

$$
\lim _{t \rightarrow \infty} y(t)=0
$$

In fact, from (1.7) and (3.53), we know that for any given $0<\epsilon<1$, there exists $\epsilon_{1}>0$ such that

$$
\begin{gathered}
\mathbf{A}_{\omega}\left(g+\sum_{i=1}^{2} h_{i} \phi_{i}\left(t,\left(x_{i}^{*}+\epsilon_{1}\right)\right)\left(x_{i}^{*}+\epsilon_{1}\right)^{2}-q \epsilon\right)>0 \\
\prod_{k=1}^{q}\left(1-u_{k}\right)<\exp \left(-\omega \mathbf{A}_{\omega}\left(g+\sum_{i=1}^{2} h_{i} \phi_{i}\left(t,\left(x_{i}^{*}+\epsilon_{1}\right)\right)\left(x_{i}^{*}+\epsilon_{1}\right)^{2}-q \epsilon\right)\right) .
\end{gathered}
$$


Note that $q(t)>0$ for $t \geq 0$. Since

$$
\begin{gathered}
\dot{x}_{1} \leq a(t) x_{2}-b(t) x_{1}-d(t) x_{1}^{2}, \\
\dot{x}_{2} \leq c(t) x_{1}-f(t) x_{2}^{2},
\end{gathered}
$$

for the given $\epsilon_{1}>0$, it is easy to show that there exists $T^{(1)}>0$ such that

$$
x_{i}(t) \leq x_{i}^{*}(t)+\epsilon_{1} \quad \text { for } t>T^{(1)}, i=1,2,
$$

we then have

$$
\prod_{k=1}^{q}\left(1-u_{k}\right)<\exp \left(-\omega \mathbf{A}_{\omega}\left(g+\sum_{i=1}^{2} h_{i} \phi_{i}\left(t, x_{i}\right)\left(x_{i}\right)^{2}-q \epsilon\right)\right) .
$$

We now show that there must exist $T^{(2)}\left(>T^{(1)}\right)$ such that $y\left(T^{(2)}\right)<\epsilon$. Otherwise, by the last two equations in system (1.4), we have

$\epsilon \leq y(t) \leq y\left(T^{(1)}\right) \prod_{T^{(1)}<\tau_{k}<t}\left(1-u_{k}\right) \exp \left(\int_{T^{(1)}}^{t}\left(g(s)+\sum_{i=1}^{2} h_{i}(s) \phi_{i}\left(s, x_{i}(s)\right) x_{i}^{2}(s)-q(s) \epsilon\right) d s\right) \longrightarrow 0$ as $t \longrightarrow \infty$.

This implies that $\epsilon \leq 0$, which is a contradiction.

Let $M(\epsilon)=\max _{t \in[0, \omega]}\left\{g(t)+\sum_{i=1}^{2} h_{i}(t) \phi_{i}\left(s, x_{i}(t)\right) x_{i}^{2}(t)+q(t) \epsilon\right\}$. Note that $M(\epsilon)$ is bounded. We then show that

$$
y(t) \leq \epsilon \exp (M(\epsilon) \omega) \quad \text { for } t \geq T^{(2)} .
$$

Otherwise, there exists $T^{(3)}>T^{(2)}$ such that $T^{(3)} \in\left(T^{(2)}+P_{1} \omega, T^{(2)}+\left(P_{1}+1\right) \omega\right]$, in which $P_{1} \in \mathbf{Z}_{+}=\{0,1,2, \ldots\}$, and $y\left(T^{(3)}\right)>\epsilon \exp (M(\epsilon) \omega)$. By (3.58), we have

$$
\begin{aligned}
\epsilon \exp (M(\varepsilon) \omega)< & y\left(T^{(3)}\right) \\
= & y\left(T^{(2)}\right) \prod_{T^{(2)}<\tau_{k}<T^{(3)}}\left(1-u_{k}\right) \exp \left(\int_{T^{(2)}}^{T^{(3)}}\left(g(s)+\sum_{i=1}^{2} h_{i}(s) \phi_{i}\left(s, x_{i}(s)\right) x_{i}^{2}(s)-q(s) \epsilon\right) d s\right) \\
\leq & y\left(T^{(2)}\right)\left[\prod_{k=1}^{q}\left(1-u_{k}\right) \exp \left(\int_{0}^{\omega}\left(g(s)+\sum_{i=1}^{2} h_{i}(s) \phi_{i}\left(s, x_{i}(s)\right) x_{i}^{2}(s)-q(s) \epsilon\right) d s\right)\right]^{P_{1}} \\
& \times \prod_{T^{(2)}+P_{1} \omega<\tau_{k}<T^{(3)}}\left(1-u_{k}\right) \exp \left(\int_{0}^{\omega}\left(g(s)+\sum_{i=1}^{2} h_{i}(s) \phi_{i}\left(s, x_{i}(s)\right) x_{i}^{2}(s)-q(s) \epsilon\right) d s\right) \\
< & \epsilon \exp (M(\varepsilon) \omega),
\end{aligned}
$$

which leads to a contradiction. This implies that (3.60) holds. In view of the arbitrariness of $\epsilon$, we get that $y(t) \rightarrow 0$ as $t \rightarrow \infty$. This completes the proof. 
Remark 3.6. From the proof of Theorem 3.1 above, we note that the predator population $y$ in system (1.4) will be extinct provided that

$$
\mathbf{A}_{\omega}\left(g+\sum_{i=1}^{2} h_{i} \phi_{i}\left(t, x_{i}^{*}\right) x_{i}^{*^{2}}\right) \leq 0,
$$

or excessive harvesting, that is,

$$
\begin{gathered}
\mathbf{A}_{\omega}\left(g+\sum_{i=1}^{2} h_{i} \phi_{i}\left(t, x_{i}^{*}\right) x_{i}^{*^{2}}\right)>0, \\
\prod_{k=1}^{q}\left(1-u_{k}\right) \leq \exp \left(-\omega \mathbf{A}_{\omega}\left(g+\sum_{i=1}^{2} h_{i} \phi_{i}\left(t, x_{i}^{*}\right) x_{i}^{*^{2}}\right)\right) .
\end{gathered}
$$

\section{Discussion}

Our result could provide a useful insight into the conservation of beneficial animals, especially rare animals. As an example, we depict the case of Oreolalax omeimontis tadpole, Oreolalax omeimontis, and red-eared slider (T. scripta elegans). Oreolalax omeimontis is a rare species of frog found near Mt. Omei in Sichuan (China). The red-eared slider is a native of the Mississippi Valley area of the United States $[8,20]$. Since the 1970s, large numbers of red-eared sliders have been produced on turtle farms in the USA for the international pet trade. Red-eared Turtles are traded as pet animals and have been introduced to many countries. They are omnivorous and will eat insects, tadpoles, crayfish, shrimp, worms, snails, amphibians and small fish, as well as aquatic plants, and they hardly may be controlled by a natural enemy. In our model, the variables $x_{1}(t)$ and $x_{2}(t)$ represent the density of Oreolalax omeimontis tadpole and Oreolalax omeimontis at time $t$, respectively. The variable $y(t)$ describes the density of the red-eared slider at time $t$. It is well known that Oreolalax omeimontis is beneficial to humans because they eat so many insect pests. Ironically, although red-eared sliders have been widely introduced throughout the world, its detrimental effects have been reported by many researchers $[21,22]$. The red-eared slider is one of main enemies of Oreolalax omeimontis. To protect these beneficial and rare toads, we must control the amount of red-eared sliders in the habitat of Oreolalax omeimontis. From a control point of view, our aim is to keep red-eared sliders at an acceptably low level with a minimum use of artificial control measures, not to eradicate all red-eared sliders. Hence, in the above example, the problem of nonextinction of populations becomes a description of reasonable harvesting rates $u_{k}(k=1,2 \ldots q)$.

According to our main result, system (1.4) is permanent if and only if the growth of the predator $y$ by foraging prey populations $x_{1}$ and $x_{2}$ plus its intrinsic rate of increase is positive on average during the period $\omega$, and harvesting rates $u_{k}(k=1,2, \ldots q)$ of red-eared sliders during the period $\omega$ are small enough to satisfy that

$$
\prod_{k=1}^{q}\left(1-u_{k}\right)>\exp \left(-\omega \mathbf{A}_{\omega}\left(g+\sum_{i=1}^{2} h_{i} \phi_{i}\left(t, x_{i}^{*}\right) x_{i}^{*^{2}}\right)\right) .
$$

These seem to be reasonable from a biological point of view; but it should be noted that condition (3.1) allows the predator to be $g+\sum_{i=1}^{2} h_{i} \phi_{i}\left(t, x_{i}^{*}\right) x_{i}^{*^{2}}<0$ for some time intervals among $0 \leq t \leq \omega$. That is, under reasonable harvesting rates, the predator can survive together with prey populations even if the growth rate of the former is negative for some seasons during a period. We hope that our result can be used to help protect beneficial animals found in their habitats. 


\section{Acknowledgment}

This work is supported by the National Natural Science Foundation of China (10671001).

\section{References}

[1] W. G. Aiello and H. I. Freedman, "A time-delay model of single-species growth with stage structure," Mathematical Biosciences, vol. 101, no. 2, pp. 139-153, 1990.

[2] K. G. Magnússon, "Destabilizing effect of cannibalism on a stage-structured predator-prey system," Mathematical Biosciences, vol. 155, no. 1, pp. 61-75, 1999.

[3] S. Neira, H. Arancibia, and L. Cubillos, "Comparative analysis of trophic structure of commercial fishery species off Central Chile in 1992 and 1998," Ecological Modelling, vol. 172, no. 2-4, pp. 233-248, 2004.

[4] J. R. Bence and R. M. Nisbet, "Space-limited recruitment in open systems: the importance of time delays," Ecology, vol. 70, no. 5, pp. 1434-1441, 1989.

[5] H. J. Barclay and P. van den Driessche, "A model for a species with two life history stages and added mortality," Ecological Modelling, vol. 11, no. 3, pp. 157-166, 1980.

[6] H. I. Freedman and J. H. Wu, "Persistence and global asymptotic stability of single species dispersal models with stage structure," Quarterly of Applied Mathematics, vol. 49, no. 2, pp. 351-371, 1991.

[7] J. M. Cushing, "Periodic time-dependent predator-prey systems," SIAM Journal on Applied Mathematics, vol. 32, no. 1, pp. 82-95, 1977.

[8] G. Krukonis and W. M. Schaffer, "Population cycles in mammals and birds: does periodicity scale with body size?" Journal of Theoretical Biology, vol. 148, no. 4, pp. 469-493, 1991.

[9] D. D. BaĬnov and P. S. Simeonov, Impulsive Differential Equations: Periodic Solutions and Applications, vol. 66 of Pitman Monographs and Surveys in Pure and Applied Mathematics, Longman Scientific and Technical, Harlow,UK, 1993.

[10] V. Lakshmikantham, D. D. Baĭnov, and P. S. Simeonov, Theory of Impulsive Differential Equations, vol. 6 of Series in Modern Applied Mathematics, World Scientific, Teaneck, NJ, USA, 1989.

[11] B. Shulgin, L. Stone, and Z. Agur, "Pulse vaccination strategy in the SIR epidemic model," Bulletin of Mathematical Biology, vol. 60, no. 6, pp. 1123-1148, 1998.

[12] J. Hui and L. Chen, "Impulsive vaccination of SIR epidemic models with nonlinear incidence rates," Discrete and Continuous Dynamical Systems. Series B, vol. 4, no. 3, pp. 595-605, 2004.

[13] B. Liu, L. Chen, and Y. Zhang, "The dynamics of a prey-dependent consumption model concerning impulsive control strategy," Applied Mathematics and Computation, vol. 169, no. 1, pp. 305-320, 2005.

[14] X. Liu and L. Chen, "Complex dynamics of Holling type II Lotka-Volterra predator-prey system with impulsive perturbations on the predator," Chaos, Solitons \& Fractals, vol. 16, no. 2, pp. 311-320, 2003.

[15] A. Lakmeche and O. Arino, "Bifurcation of non trivial periodic solutions of impulsive differential equations arising chemotherapeutic treatment," Dynamics of Continuous, Discrete and Impulsive Systems, vol. 7, no. 2, pp. 265-287, 2000.

[16] S. Tang and L. Chen, "Density-dependent birth rate, birth pulses and their population dynamic consequences," Journal of Mathematical Biology, vol. 44, no. 2, pp. 185-199, 2002.

[17] E. Funasaki and M. Kot, "Invasion and chaos in a periodically pulsed mass-action chemostat," Theoretical Population Biology, vol. 44, no. 2, pp. 203-224, 1993.

[18] J. Cui, L. Chen, and W. Wang, "The effect of dispersal on population growth with stage-structure," Computers \& Mathematics with Applications, vol. 39, no. 1-2, pp. 91-102, 2000.

[19] V. Lakshmikantham, V. M. Matrosov, and S. Sivasundaram, Vector Lyapunov Functions and Stability Analysis of Nonlinear Systems, vol. 63 of Mathematics and Its Applications, Kluwer Academic Publishers Group, Dordrecht, The Netherlands, 1991.

[20] P. C. H. Pritchard, Living Turtles of the World, T.F.H. Publications, Neptune, NJ, USA, 1967.

[21] T. Chen and K. Lue, "Ecological notes on feral populations of Trachemys scripta elegans in northern Taiwan," Chelonian Conservation and Biology, vol. 3, no. 1, pp. 87-90, 1998.

[22] L. M. Close and R. A. Seigel, "Differences in body size among populations of red-eared sliders (Trachemys scripta elegans) subjected to different levels of harvesting," Chelonian Conservation and Biology, vol. 2, no. 4, pp. 563-566, 1997. 


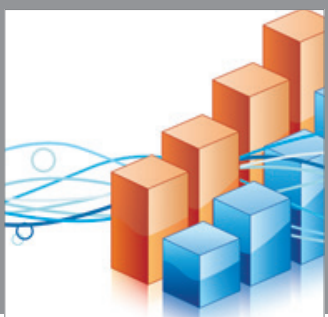

Advances in

Operations Research

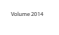

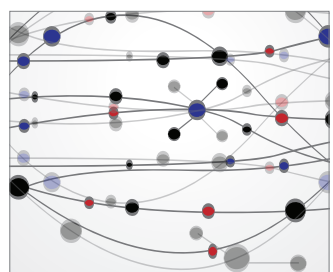

\section{The Scientific} World Journal
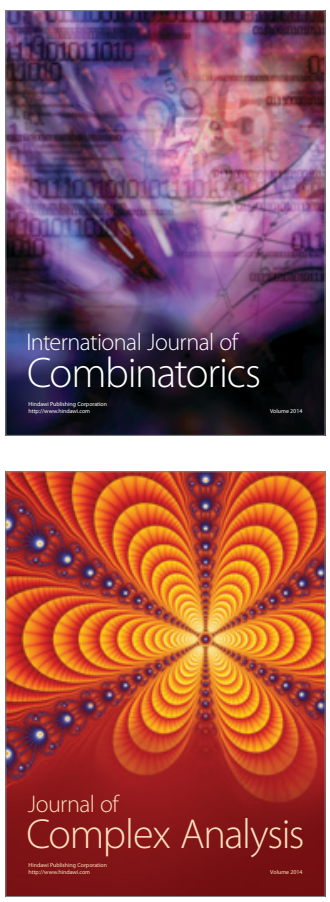

International Journal of

Mathematics and

Mathematical

Sciences
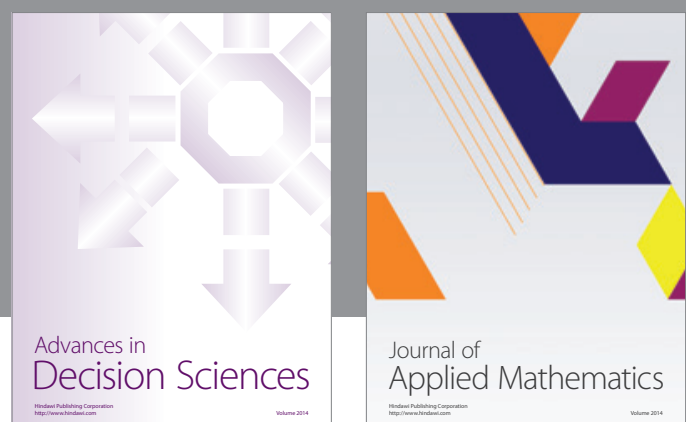

Journal of

Applied Mathematics
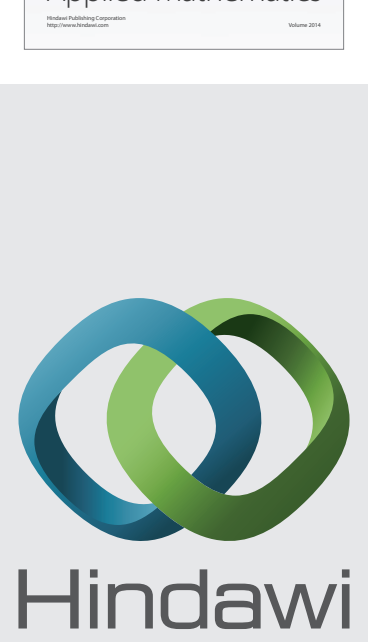

Submit your manuscripts at http://www.hindawi.com
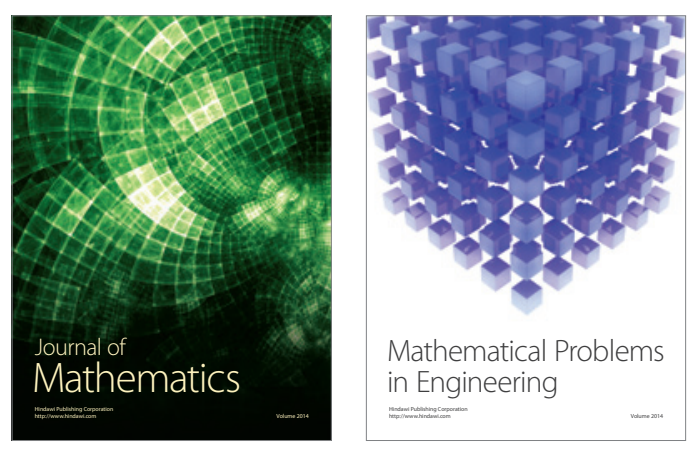

Mathematical Problems in Engineering
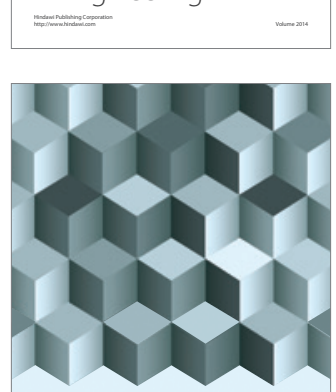

Journal of

Function Spaces
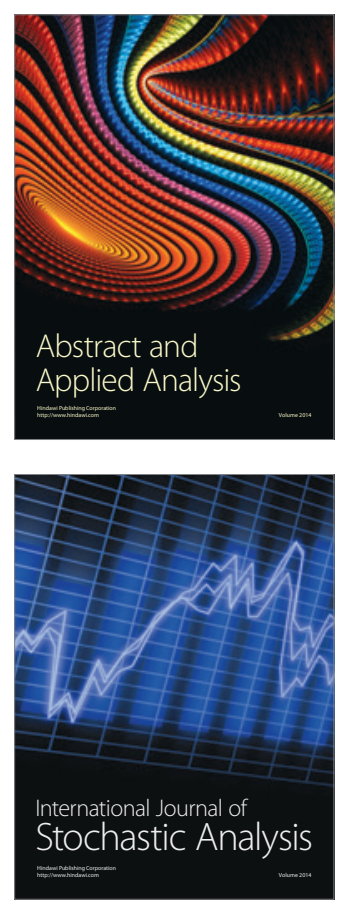

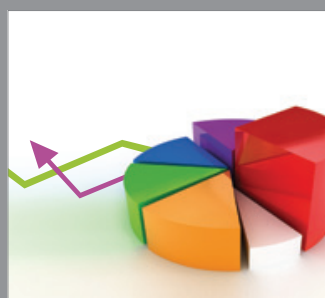

ournal of

Probability and Statistics

Promensencen
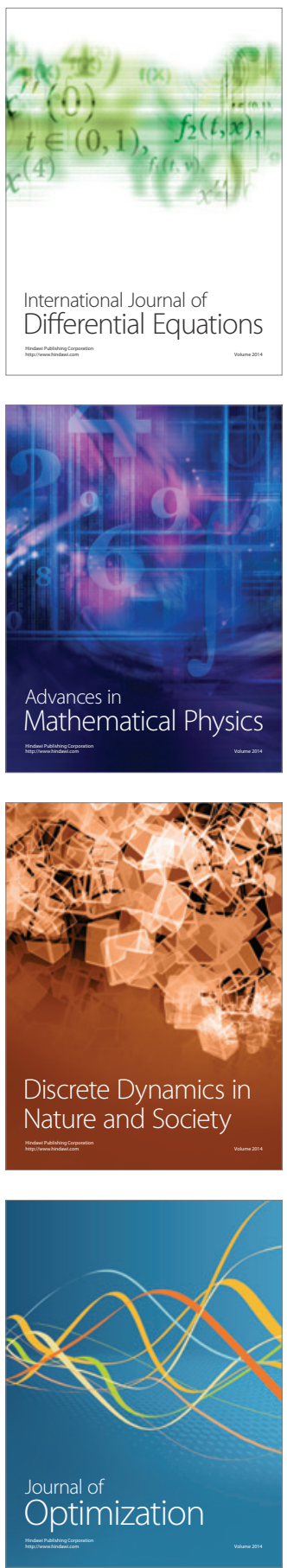